\title{
Descripción de la enfermedad de von Hippel-Lindau en tres familias españolas
}

\author{
K. VILLAR GÓMEZ DE LAS HERAS \\ Centro de Salud de Navahermosa. Toledo
}

\author{
DESCRIPTION OF THE VON HIPPEL-LINDAU DISEASE IN THREE \\ SPANISH FAMILIES
}

\section{RESUMEN}

Objetivo: Describir la evolución de la enfermedad y valorar su seguimiento clínico en 10 pacientes de tres familias españolas.

Pacientes y métodos: Los pacientes fueron localizados a través de la Alianza de Familias de von Hippel-Lindau de EEUU (VHLFA). Se recopilaron todos los informes médicos y se realizó entrevista personal en el mes de Mayo de 2001. Estudio retrospectivo de una serie de casos.

Resultados: La historia familiar era sugerente de enfermedad de von Hippel-Lindau en dos de las familias. En la tercera se describe una mutación "de novo". La edad media es de 30,8 años (rango: 15-53). La edad de aparición de los primeros síntomas fue inferior a 30 años en 9 pacientes. 8 debutaron con afectación visual. Se observan hemangioblastomas del SNC en 7 pacientes. La mayoría no seguía ningún protocolo coordinado de las diferentes especialidades implicadas, 6 tenían afectación pancreática documentada y 3 cáncer renal. Hay constancia de despistaje de feocromocitoma en 5 pacientes. 4 habían fallecido por la evolución de la enfermedad. El estudio genético se había realizado en 4 de los 6 supervivientes.

Conclusiones: La enfermedad en nuestra serie sigue una evolución similar a la descrita en la literatura. El diagnóstico ha sido tardío en dos de las familias. El seguimiento ha sido deficiente y puede haber condicionado la morbimortalidad.

PALABRAS CLAVE: Enfermedad de von Hippel- Lindau. Hemangioblastoma. Feocromocitoma. Carcinoma renal.

\begin{abstract}
Purpose: To describe disease evolution and clinical follow-up of 10 patients in three Spanish families.

Patients and methods: Patients were contacted through the von Hip pel-Lindau Family Alliance (VHLFA). Compilation of medical reports and patient interviews were carried out in May of 2001. This is a retros pective study of a case series.

Results: Family history was suggestive of von Hippel-Lindau disease in two of the families. A 'de novo' mutation is described in the third family. The mean patient age is 30,8 (range: 15-53). The age of presen tation was $<30$ in 9 patients. In 8 of the patients, eye involvement was the first VHL presentation. 7 patients currently have hemangioblastomas of the CNS. Most patients are not participants in management protocols for the coordinate care of their various VHL lesions. 6 patients had documented pancreatic involvement and 3 patients had renal cancer. Clinical screening for occult pheochromocytomas were performed in 5 pacients. 4 were deceased from VHL-related complications. Genetic stu dies had been performed in 4 of the 6 survivors.

Conclusions: Disease progression in our series is similar to that pre viously described for other families. Diagnosis was delayed in two of the families. Suboptimal patient management may have contributed to the high morbidity and mortality.
\end{abstract}

KEY WORDS: von Hippel-Lindau disease. Hemangioblastoma, pheoch romocytoma. Carcinoma, renal cell.

\section{INTRODUCCIÓN}

La enfermedad de von Hippel-Lindau es un proceso de herencia autosómica dominante, ocasionado por la mutación del gen oncosupresor vHL (brazo corto del cromosoma 3). Esta mutación predispone al desarrollo de tumores en diferentes órganos: hemangioblastomas (HB) en el SNC y retina, quistes y carcinoma renal de células claras, feocromocitomas, quistes y tumores neuroendocrinos pancreáticos, tumores de saco endolinfático (ELST) y cistoadenomas en epidídimo y anejos femeninos. La mayoría son multicéntricos o bilaterales, y aparecen a edades más tempranas que en los no portadores de la mutación $(1,2,3,4,5,17)$.

La prevalencia es de al menos 1/36.000 (2). Las primeras manifestaciones aparecen a cualquier edad, pero frecuentemente los pacientes presentan el primer problema entre los 20 y los 30 años (6), y habitualmente consisten en síntomas neurológicos o alteraciones del campo visual (3). La penetrancia es incompleta pero elevada en los portadores de la mutación, de forma que casi todos han desarrollado tumores característi-

Trabajo aceptado: 30 de abril de 2002

Correspondencia: Karina Villar Gómez de las Heras. C/ Virgen del Rosario, 30.45280 Olías del Rey (Toledo). e-mail: kareen@jazzcyber.com / myoxam@yahoo.com 
cos a la edad de 60 años $(2,8)$. La esperanza media de vida era inferior a 50 años antes de instaurarse los actuales protocolos de seguimiento, que deben comenzarse en la infancia $(3,6)$. Actualmente las metástasis del carcinoma renal y las complicaciones neurológicas de los HB cerebelosos son las causas más comunes de muerte (3). El pronóstico depende en gran medida de la detección y tratamiento tempranos de estos tumores $(2,3,4)$. El mayor problema pronóstico surge cuando el paciente es el primer caso dentro de la familia (mutación "de novo") y la enfermedad permanece sin diagnosticar mucho más tiempo. La localización del gen se basa en la búsqueda de marcadores genéticos que muestran la pérdida de la heterozigosidad en el tejido tumoral, según el modelo de la doble alteración de Knudson (10), que implica una segunda mutación somática que afecta al alelo normal de los genes supresores de tumores. El análisis del ADN y la identificación de los portadores para seguimiento desde temprana edad se perfilan como la mejor prevención de las complicaciones $(7,17)$. Debe realizarse paralelamente el estudio del árbol genealógico familiar. Aquellos miembros de la familia que se compruebe que no son portadores de la mutación, no precisan someterse a ningún otro tipo de pruebas.

La enfermedad no tiene una correlación clara genotipofenotipo ni siquiera en gemelos idénticos $(11,12)$, por lo que se siguen estudiando las variantes cromosómicas en relación con las características fenotípicas de los pacientes.

El objetivo del presente trabajo es describir la evolución de la enfermedad y el seguimiento realizado hasta el momento en tres familias españolas.

\section{PACIENTES Y MÉTODOS}

Describimos las manifestaciones de la enfermedad en 10 de los miembros afectos de tres familias de diferentes regiones de la geografía española (dos de Barcelona y una de Toledo, sin ancestros comunes en las cuatro últimas generaciones), a partir de la revisión de los informes médicos y entrevista personal. 4 pacientes habían fallecido como consecuencia de la evolución de la enfermedad. Sólo uno seguía revisiones coordinadas por un facultativo, mientras que los demás habían cambiado de especialistas varias veces, por lo que se les pidió que solicitaran copia de todos sus informes en los diferentes hospitales. Se recuperaron los informes de las autopsias de 2 de los fallecidos.

Las familias fueron localizadas a través de la VHL Family Alliance (www.vhl.org). La búsqueda bibliográfica se realizó en Medline sin fecha límite retrospectiva de publicación y hasta Mayo de 2001. Las palabras clave utilizadas fueron: enfermedad de von Hippel-Lindau, hemangioblastoma, cáncer renal, feocromocitoma.

\section{RESULTADOS}

El árbol genealógico revela una historia de varias generaciones de afectados en dos de las familias, mientras que en la tercera hay un único caso (Fig. 1). La evolución de la enfermedad en cada persona queda representada en la figura 2 , y el seguimiento que se ha hecho hasta la fecha de recogida de los datos está reflejado en la tabla I.

Los HB retinianos fueron la forma más frecuente de presentación de la enfermedad (8). En el caso 10 existe un angioma único localizado en la papila del nervio óptico del ojo izquierdo, mientras que en los demás pacientes los tumores han sido múltiples (desde 2 que ha presentado hasta ahora la paciente 9 , a los 14 de la paciente 3). Los tratamientos más utilizados son la fotocoagulación con láser y la crioablación. A pesar del tratamiento, 3 pacientes sufrieron amaurosis unilateral y pérdida importante de visión en el otro ojo ( $\operatorname{casos} 1,4$ y 6 ).

La afectación del SNC se observa en 8 pacientes. El caso 5 tiene HB múltiples a nivel medular y su sintomatología es característica de esta forma de manifestación de la enfermedad (8). Curiosamente no tenía HB intracraneales en la última exploración (1999). En los restantes casos, los HB cerebelosos han sido múltiples -característico de la enfermedad $(3,7,17)$.

Seis pacientes han sido diagnosticados de afectación pancreática mediante ecografía, TAC abdominal, o por autopsia. 4 tienen quistes múltiples y 1 una tumoración polilobulada sin estudios posteriores (caso 9). El sexto paciente (caso 2) falleció de un tumor neuroendocrino pancreático.

Tenemos constancia de diagnóstico de cáncer renal en tres pacientes (casos 1, 3 y 5). El despistaje de feocromocitoma sólo se ha realizado en una o dos ocasiones mediante determinación de catecolaminas en orina de 24 horas, complementado con TAC abdominal en 2 pacientes (casos 3 y 10). Los resultados fueron negativos salvo en el caso 3 , actualmente en estudio.

Las causas de fallecimiento fueron: tumor neuroendocrino de origen no filiado (caso 1), tumor neuroendocrino pancreático (caso 2) y complicaciones postquirúrgicas de los $\mathrm{HB}$ cerebelosos ( $\operatorname{casos} 4$ y 7).

El estudio genético del ADN se ha realizado en los pacientes actualmente vivos de las familias A y $\mathrm{C}$. En la rama estudiada de la familia $\mathrm{B}$ faltaba realizar el estudio genético a dos de los hijos del paciente 5, de 10 y 12 años de edad, y a uno de la paciente 4 (asintomático). Ninguno de los fallecidos tenía el análisis genético (el gen responsable de la mutación se localizó por primera vez en 1993 y todos salvo el caso 4 fallecieron antes de dicha fecha). En la familia $\mathrm{C}$ se realizó el estudio genético a padres y hermanos, pensando en la posibilidad de una mutación en la línea germinal de alguno de los progenitores, condicionada por un mosaicismo. En los últimos años muchas de las pruebas solicitadas han sido demandadas por los propios pacientes, que se han informado sobre su enfermedad a través de Internet.

\section{DISCUSIÓN}

Los datos clínicos obtenidos concuerdan con lo publicado hasta el momento sobre la evolución típica de la enfermedad, aunque no hay acuerdo en la secuencia de presentación de la sintomatología. En nuestra serie la clínica oftalmológica es la forma más frecuente de presentación, y la afectación neurológica sigue en secuencia a la anterior, con la aparición de HB preferentemente a nivel cerebeloso. Algunos autores refieren una cronología similar (1), mientras otros han registrado una presentación a nivel del SNC, y posteriormente oftalmológica $(4,7)$.

En la literatura se ha descrito la afectación medular ligada a la enfermedad hasta en un $50 \%$ de los pacientes, con un número considerable de casos asintomáticos $(8,17)$. Sin embargo, en nuestra serie sólo 1 caso registra esta localización, lo que podría explicarse por la tendencia a no realizar resonancia magnética medular como parte del seguimiento. Dado que el crecimiento de los tumores es muy lento, y la edad media de nuestra serie ronda los 30 años, cabe la posibilidad de HB incipientes a nivel medular. 
354

K. VILLAR GÓMEZ DE LAS HERAS

AN. MED. INTERNA (Madrid)
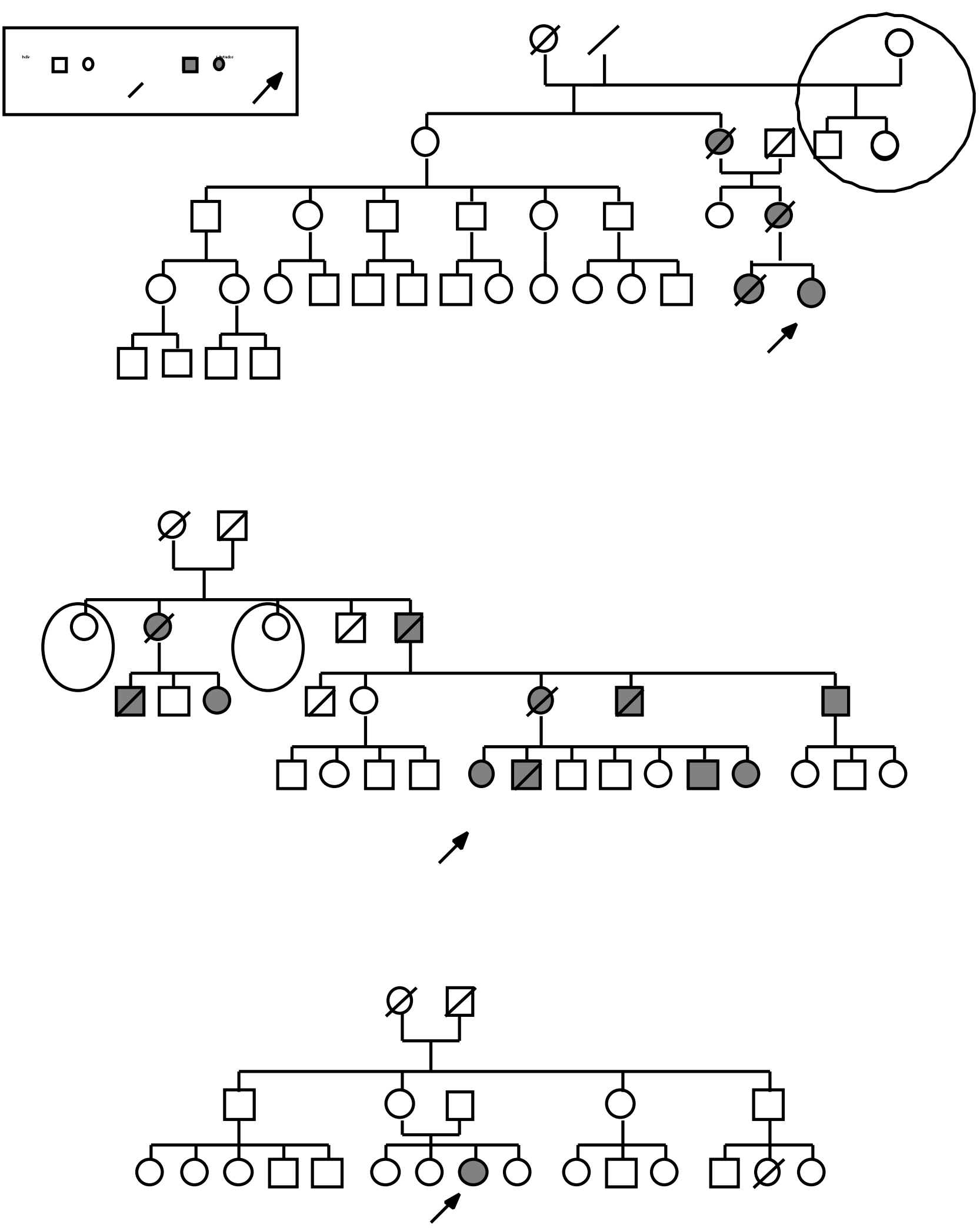

Fig. 1. Árboles genealógicos de las tres familias. 


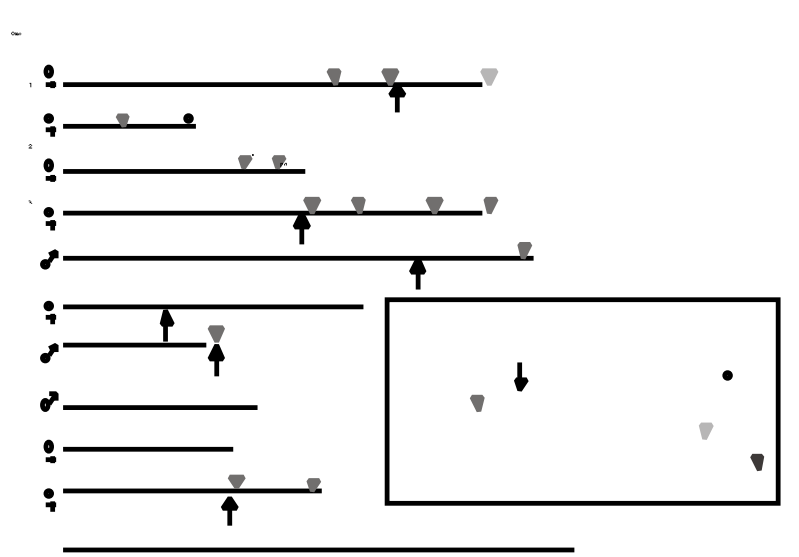

Fig. 2. Evolución de las manifestaciones clínicas en nuestra serie.

Contrasta la aparente temprana edad de aparición de HB retinianos en la familia $\mathrm{B}$ con los datos de otros estudios, en los que se registra una edad media de aparición entorno a los 25 años $(1,3)$. Podría explicarse por la preocupación personal de los afectados de esta familia en hacerse revisiones oftalmológicas desde la adolescencia, cuando los tumores eran muy pequeños y por tanto asintomáticos. Para poder establecer comparaciones sería necesario conocer las circunstancias de detección de hemangioblastomas retinianos en otras series.

Observamos afectación pancreática en 6 de los 10 casos, aunque igualmente puede ser una cifra sólo aproximada debido a la falta de estudios seriados de imagen. Algunos autores han registrado prevalencias del 15-40\%(4), mientras que Hammel y cols., informaron de un $77 \%$, similar a lo encontrado por Horton y cols., en una serie de autopsias de 29 pacientes con enfermedad de VHL(13). Estas diferencias pueden deberse una vez más a la existencia de lesiones latentes durante largos periodos de tiempo, que se descubren bien por screening sistemático de pacientes VHL, bien de forma accidental.

Sorprende el hecho de que no se haya realizado estudio de la tumoración pancreática de la paciente 9 , pues aun cuando los tumores neuroendocrinos son una entidad poco frecuente, en muchas ocasiones son hipofuncionantes y la sintomatología puede ser mínima. La paciente 2 merece mención especial, pues por la afectación que presentaba (síndrome de Cushing a los 5 años, adenoma cromófobo hipofisario a los 7 y tumor neuroendocrino pancreático a los 15), parece más una neoplasia endocrina múltiple (MEN) tipo 1 que una enfermedad de von Hippel-Lindau. Actualmente se está realizando el estudio genético tanto para VHL como para MEN de la familia A (a partir de muestras de necropsia).

El cáncer renal aparece normalmente alrededor de los 40 años en los afectados por la mutación del gen vHL, lo que explica que sólo haya 3 pacientes diagnosticados hasta ahora. Ha sido interesante disponer del informe de la autopsia de la paciente 1 , pues el cáncer que le causó finalmente la muerte fue un tumor neuroendocrino metastásico.

Aunque el feocromocitoma ha sido descrito asociado a la enfermedad en un 10-25\% de los casos (4), no se realiza despistaje, ni siquiera como parte del estudio preoperatorio de estos pacientes -contrariamente a lo que recomiendan los expertos (8)- lo que podría estar condicionando la morbilidad perioperatoria en la población VHL. En nuestra serie sólo está registrada su realización previa a la cirugía en 1 caso (10).

Otras manifestaciones típicas de VHL no aparecen recogidas en los informes médicos. Es difícil estimar la frecuencia de tumor de saco endolinfático, o cistoadenomas epididimarios o

\section{TABLA I}

PRUEBAS CO M PLEM ENTARIAS REALIZADAS HASTA EL M O M ENTO, DESDE EL HALLAZGO DE LA PRIM ERA LESIÓ N DE CADA Ó RGANO. SE HAN EXCLUIDO LOS PACIENTES YA FALLECIDOS PO R DISPO NER DE ESCASA INFO RM ACIÓ N ESCRITA

\begin{tabular}{|c|c|c|c|c|c|c|}
\hline Caso & Edad & $\begin{array}{l}\text { Revisiones en } \\
\text { oftalmología }\end{array}$ & $\begin{array}{l}\text { Revisiones en } \\
\text { neurocirugía }\end{array}$ & $\begin{array}{c}\text { Exploraciones } \\
\text { imagen abdominal }\end{array}$ & $\begin{array}{c}\text { Catecolaminas } \\
\text { orina } 24 \mathrm{~h}\end{array}$ & $\begin{array}{l}\text { Diagnóstico } \\
\text { genético }\end{array}$ \\
\hline 3 & 26 & Cada 6-12 meses & $\begin{array}{l}\text { RM N craneal anual } \\
\text { RM N medular } 1996\end{array}$ & TAC 1996,2001 & 2001 & Londres 2000 \\
\hline 5 & 53 & Anualmente & $\begin{array}{c}\text { TAC craneal } 1985 \\
\text { RM N craneal y } \\
\text { cervical en } 1999\end{array}$ & TAC 1999, 2000 & No & No \\
\hline 6 & 33 & Cada 6-12 meses & $\begin{array}{c}\text { TAC craneal en 1998, } 99 \\
\text { y } 2001\end{array}$ & $\begin{array}{c}\text { TAC } 1998 \\
\text { Ecografía } 1999\end{array}$ & 2000 y 2001 & $\begin{array}{c}\text { H. Clínico } \\
\text { Barcelona } 1998\end{array}$ \\
\hline 8 & 23 & Cada 6-12 meses & TAC craneal 1998, 99 & $\begin{array}{c}\text { TAC } 1998 \\
\text { Ecografía } 1999\end{array}$ & 2001 & $\begin{array}{c}\text { H. Clínico } \\
\text { Barcelona } 1998\end{array}$ \\
\hline 9 & 19 & Cada 3-6 meses & TAC craneal 1999 & TAC 2000 & 2001 & No \\
\hline 10 & 27 & Cada 6-12 meses & $\begin{array}{l}\text { RM N craneal anual } \\
\text { RM N cervical } 2000\end{array}$ & $\begin{array}{l}\text { Eco cada 6-12 meses } \\
\text { TAC 1998, } 2001\end{array}$ & 2000 y 2001 & $\begin{array}{c}\text { Fundación } \\
\text { Jiménez-Díaz } \\
1998\end{array}$ \\
\hline
\end{tabular}


en anejos femeninos en pacientes asintomáticos, como ocurre en la serie presentada.

En todos los pacientes salvo en uno (caso 10) se observa un deficiente seguimiento, pues no se han coordinado las pruebas complementarias necesarias para diagnosticar precozmente ni los HB, ni los tumores pancreáticos y renales, aun cuando queda de manifiesto que -como está descrito en la literatura- son causa importante de morbilidad y mortalidad. Varios autores han propuesto protocolos de seguimiento similares $(1,2,4,6)$, consensuados en el último simposio sobre VHL (14).

El diagnóstico de enfermedad de von Hippel-Lindau no suele hacerse en un primer momento, pues no suele considerar se el síndrome en pacientes con $\mathrm{HB}$ del $\operatorname{SNC}(7,8)$ - a pesar de que alrededor de un $30 \%$ lo tienen $(3,6,9)$. Un alto porcentaje de HB en menores de 30 años se asocia a VHL (60\% en la serie de Richard y cols., 1994)(4). Gläsker y cols. recomiendan análisis del ADN en todo paciente con HB del SNC $(7,9)$.

Las familias B y C tenían recogido el árbol genealógico al menos en parte (la B en la historia de Medicina Interna y la C en la de Genética). Observando éste se puede llegar a determinar el antecesor transmisor de la mutación y las ramas familiares por las que se ha extendido. La búsqueda activa de familiares afectos a partir del caso índice, sólo se había llevado a cabo entre los familiares hasta de segundo grado colateral. Esto hace sospechar la existencia de otros afectados no identificados en el estudio, algunos de los cuales todavía son jóvenes y no han dado manifestaciones.

$\mathrm{El}$ caso de la familia $\mathrm{C}$ se debe casi con toda seguridad a una mutación "de novo" -producida probablemente durante la embriogénesis-. Cabría la posibilidad de un mosaicismo parental (la mutación se produce en el progenitor en etapas tardías de la embriogénesis, de forma que tiene líneas celulares mutadas y otras no), con lo que el estudio genético puede dar un resultado falsamente negativo (15). Sin embargo esto es muy improbable, pues los estudios clínicos realizados (RMN de SNC y fondo de ojo), fueron negativos a una edad a la que normalmente la enfermedad ya se ha manifestado -tenían ambos alrededor de 55 años.

La enfermedad de VHL en nuestra serie sigue una evolución similar a la descrita en otras series de la literatura. El diagnóstico como tal ha sido tardío por desconocimiento de la misma, y no se han relacionado entre sí los casos familiares hasta años después del primer síntoma. Aun entonces no se ha considerado el análisis genético como medio para descartar otros casos en la misma familia. La mayoría de los pacientes son seguidos en diferentes servicios sin una coordinación entre ellos y sin un protocolo de seguimiento, lo que puede estar condicionando en gran medida la morbimortalidad. Llama la atención que la sintomatología a la que más cuidados se le presta es aquella que no condiciona la supervivencia del paciente: la oftalmológica. La información que aportan las autopsias es muy valiosa para caracterizar una enfermedad tan desconocida, por lo que debería realizarse en todos los casos.

A la vista de los trabajos publicados, podemos concluir que muchos de los casos que se están tratando actualmente como formas esporádicas de $\mathrm{HB}$, cáncer renal o pancreático, podrían ser en realidad manifestaciones de una enfermedad de VHL no diagnosticada. Los servicios de Neurocirugía y Oftalmología tienen una especial responsabilidad en el diagnóstico inicial de estos pacientes. La serie expuesta refleja la variabilidad fenotípica característica, incluso dentro de una misma familia, hecho constatado en numerosos estudios (16).

\section{AGRADECIMIENTOS}

La realización de este trabajo ha sido posible gracias a la inestimable colaboración de todos los afectados, así como a la VHL Family Alliance, que nos puso en contacto.

\section{Bibliografía}

1. Hes FJ. Von Hippel Lindau Disease: clinical and genetic investigations in the Netherlands. Tesis, Utrecht; 2000.

http://www.library.uu.nl/digiarchief/dip/diss/1898214/inhoud.htm

2. Neumann HP. Von Hippel Lindau Disease: A Model Project. Prevention and preventive intervention in a hereditary tumor syndrome. (http://www.vhl.org/profinfo/prevention.pdf). 1998.

3. Niemelä M. Hemangioblastomas of the Central Nervous System and Retina Impact of von Hippel Lindau Disease. Tesis, Helsinki; 2000. http://ethesis.helsinki.fi/julkaisut/laa/kliin/vk/niemela/

4. Richard S, Beigelman C, Gerber S, et al. L'hémangioblastome existe-til en dehors de la maladie de von Hippel Lindau?. Neurochirurgie 1994; 40: $145-54$.

5. Neumann H, Berger D, Sigmund G, et al . Pheochromocytomas, multiple endocrine neoplasia type 2, and von Hippel- Lindau disease. N Engl J Med 1993; 329: 1531-8.

6. Friedrich CA. Von Hippel Lindau disease, a pleomorphic condition. American Cancer Society 1999; 86 (8 Supl): 1658-62.

7. Gläsker S, Bender BU, Apel TW, et al. The impact of molecular genetic analysis of the VHL gene in patients with haemangioblastomas of the central nervous system. J Neurol Neurosurg Psychiatry 1999;67:758-762.

8. Emery E, Hurth M, Lacroix-Jousselin C, David PH, Richard S. Les hémangioblastomes intra-rachidiens. A propos d'une série récente de 20 cas. Neurochirurgie 1994; 40: 165-173.

9. Hes FJ, Mckee S, Taphoorn MJB, et al. Cryptic VHL disease: germline mutations in patients with hemangioblastoma only. J Med Genet

2000; 37: 939-43 (December).

10. Knudson AG. Hereditary cancer: two hits revisited. J Cancer Res Clin Oncol 1996; 122: 135-40.

11. Wesolowski DP, Ellwood RA, Schwab RE, Farah J. Hippel-Lindau syndrome in identical twins. Case reports. British Journal of Radiology 1981; 54: 982-6.

12. Sobottka SB, Frank S, Hampl M, Schackert HK, Schackert G. Multiple intracerebral haemangioblastomas in identical twins with von Hippel Lindau disease - a clinical and molecular study. Acta Neurochir (Wien) 1998; 140: 281-5.

13. Hammel P, Vilgrain V, Terris B, et al. Pancreatic involvement in von Hippel-Lindau disease. Gastroenterology 2000; 119: 1087-95.

14. Fourth International Symposium on von Hippel-Lindau, July 20-23, 2000. Mayo Clinic. Rochester, Minnesota. http://www.vhl.org/conf2000/index.html

15. Sgambati MT, Stolle C, Choyke PL, et al. Mosaicism in von Hippel-Lindau disease: lessons from kindreds with germline mutations identified in offspring with mosaic parents. Am J Genet 2000; 66: 84-91.

16. Webster AR, Richards FM, Macronald FE, Moore AT, Maher ER. An analysis of phenotypic variation in the familial cancer syndrome von Hippel-Lindau disease: evidence for modifier effects. Am J Hum Genet 1998; 63: 1025-35.

17. Conway JE, Chou D, Clatterbuck RE, et al. Hemangioblastomas of the central nervous system in von Hippel-Lindau syndrome and sporadic cases. Neurosurg 2001; 48: 55-62. 